\title{
A novel role for sphingolipid intermediates in activation-induced cell death in $T$ cells
}

\author{
JC Solomon ${ }^{1,2}$, K Sharma $^{1}$, LX Wei ${ }^{4}$, T Fujita $^{3}$ and YF Shi ${ }^{*}{ }^{1,2,4}$ \\ 1 Department of Immunology, Holland Laboratory, American Red Cross, 15601 \\ Crabbs Branch Way, Rockville, MD 20855, USA \\ 2 Institute for Biomedical Sciences, George Washington University, Ross 664, \\ 2300 Eye St., NW, Washington, DC 20037, USA \\ ${ }^{3}$ Graduate School of Pharmaceutical Sciences, Kyoto University, Sakyo-ku, \\ Kyoto 606-01, Japan \\ ${ }^{4}$ Department of Molecular Genetics, Microbiology and Immunology, University \\ of Medicine and Dentistry of New Jersey, Robert Wood Johnson Medical \\ School, 661 Hoes Lane, Piscataway, NJ 08854, USA \\ * Corresponding author: Y Shi, Department of Molecular Genetics, \\ Microbiology and Immunology, Robert Wood Johnson Medical School, \\ University of Medicine and Dentistry of New Jersey, 661 Hoes Lane, \\ Piscataway, NJ 08854, USA. Tel: +1 732235 4501; Fax: +1 732235 4505; \\ E-mail: shiyu@umdnj.edu
}

Received 8.2.02; revised 8.8.02; accepted 21.8.02 Edited by DR Green

\begin{abstract}
Activation-induced cell death (AICD), a process mediated by CD95 and CD95 ligand (CD95L), plays a critical role in regulating homeostasis of the immune system. Although the role of sphingolipids such as ceramides has been suggested to participate in CD95-mediated apoptosis, the exact role of these molecules in this process remains controversial. We employed myriocin, a specific inhibitor of serine palmitoylCoA transferase that mediates the first commitment step in sphingolipid synthesis. We found that myriocin could effectively block AICD in T-cell hybridomas and T-cell blasts. However, fumonisin B1, an inhibitor of the final step of ceramide synthesis, or inhibitors of sphingomyelinases did not prevent AICD. Furthermore, ceramide analogues, such as C2 and C6, could not reverse the inhibitory effect of myriocin. Interestingly, sphinganine, an intermediate of ceramide synthesis, completely reversed the inhibitory effect of myriocin, indicating a critical role of sphinganine. Myriocin did not modulate the expression of CD95 or CD95L, instead, it interfered with the early steps of CD95-mediated caspase activation. Therefore, we have uncovered a novel mechanism by which sphingolipid intermediates regulate CD95-mediated apoptosis. Cell Death and Differentiation (2003) 10, 193-202. doi:10.1038/ sj.cdd. 4401136
\end{abstract}

Keywords: apoptosis; T lymphocytes; lipid mediators; cellular activation

Abbreviations: AICD, activation-induced cell death; Lpr, lymphoproliferation; gld, generalized lymphoproliferative disease; ALPS, autoimmune lymphoproliferative syndrome; DISC, deathinducing signaling complex

\section{Introduction}

One of the best-characterized systems in which apoptosis can be demonstrated is activation-induced cell death (AICD) in T cells. ${ }^{1,2}$ AICD is a fundamental mechanism for the removal of excess peripheral lymphocytes after mounting an immune response. $^{3-7}$ Defects in this programmed removal mechanism could, therefore, lead to the accumulation of potentially autoreactive lymphocytes. Lymphoproliferation (Ipr) and generalized lymphoproliferative disease $(g / d)$ are spontaneous mutations in mice that are loss of function mutations of Fas/CD95 and FasL/CD95L, respectively. ${ }^{8}$ Mice with Ipr and gld mutations produce large amounts of autoantibodies and develop an autoimmune disease that resembles human systemic lupus erythematosus. ${ }^{9}$ Autoimmune lymphoproliferative syndrome (ALPS) pathogenesis in humans has been attributed to defective lymphocyte apoptosis caused by mutations of either the CD95 or CD95L gene.

Studies in the last few years have revealed that CD95 and CD95L play a critical role in AICD. It has been demonstrated that blocking the interaction between CD95 and CD95L could inhibit AICD. ${ }^{10}$ The unique 'death domain' motif in the cytoplasmic region of the CD95 receptor is essential for the initiation of receptor-mediated caspase activation cascade. The binding of CD95L to CD95 induces trimerization, which brings together the intracellular death domains, leading to the recruitment of Fas-associated death domain (FADD). ${ }^{7}$ The death effector domain in FADD further recruits procaspase 8 (FLICE) to form the death-inducing signaling complex (DISC). ${ }^{11}$ Upon joining the complex, procaspase 8 is cleaved and becomes activated. Activated caspase 8 initiates the activation of a caspase cascade that includes caspase 3 (CPP32). Caspases can also be activated in response to cellular stress, which often targets mitochondria. Such stimuli result in the release of proapoptotic molecules including apoptosis-inducing factor (AIF) and cytochrome $c$. Once released into the cytosol, cytochrome $c$ interacts with Apaf-1, dATP/ATP and procaspase 9 to form a complex called the 'apoptosome' that can activate procaspase $9 .^{11}$ Caspase- 9 can signal downstream and activate procaspase 3 and procaspase 7. In any case, both pathways lead to the activation of caspase 3. Caspase 3 has been considered as an effector caspase, which then cleaves the inhibitor of caspase-activated deoxyribonuclease (ICAD) to release CAD. ${ }^{12}$ Once CAD is released, it degrades genomic DNA at the internucleosomal regions and ultimately leads to irreversible apoptosis.

Although the blueprints of the pathways by which CD95 induces apoptosis have been established, the details of the molecular mechanisms are yet to be revealed. One example is the role of sphingolipids in CD95-mediated apoptosis. These lipids are a large class of membrane lipids that have attracted much attention in the recent years because of their diverse roles in biological processes such as cell survival, 
Palmitoyl CoA + Serine

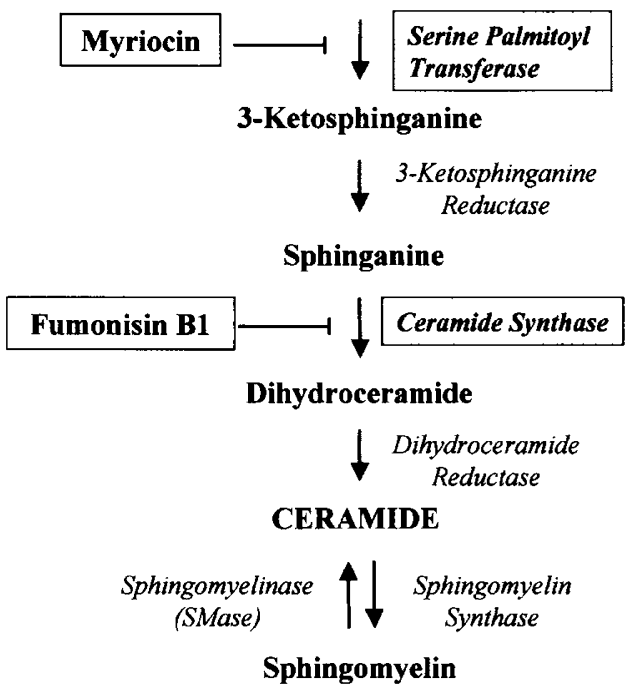

Figure 1 Diagrammatic representation of the pathways of sphingolipid biosynthesis. The framed lettering shows the target enzymes and their inhibitors

differentiation and cell death. Sphingolipids are derivatives of the C18 amino alcohols sphingosine and sphinganine. Ceramide is the fundamental structural unit common to all sphingolipids and is one of the most studied members of the sphingolipid family. The attachment of different structures to ceramide via $-\mathrm{OH}$ on $\mathrm{C}-1$ of sphingosine forms various types of sphingolipids. The first step in de novo synthesis of ceramide begins with the condensation of palmitoyl CoA with serine to form 3-ketosphinganine, which is catalyzed by serine palmitoyl transferase. 3-Ketosphinganine is subsequently reduced to form sphinganine, then acylated by ceramide synthase to yield dihydroceramide. Finally, dihydroceramide is oxidized to yield ceramide, where a trans- 4,5 double bond is introduced by the enzyme dihydroceramide reductase. ${ }^{13}$ Ceramide may also be produced via the action of several distinct enzymes involved in the cleavage of sphingomyelin (ceramide linked to phosphocholine) by acidic (a) or neutral (n) sphingomyelienases (SMases) (a diagrammatic representation of sphingolipid metabolism is shown in Figure 1). There exist two forms of nSMase: one is cytosolic, $\mathrm{Mg}^{2+}$ independent and the other is membrane-bound, $\mathrm{Mg}^{2+}$. dependent. ${ }^{14,15}$ Depending on the cell lines and experimental procedures employed, ceramide has been shown to mediate apoptosis, ${ }^{16}$ cell cycle arrest, ${ }^{17}$ induction of the JNK and ERK pathway, ${ }^{18}$ increased cell survival, ${ }^{19}$ cell proliferation ${ }^{20}$ and activation of $\mathrm{NF}_{\kappa} \mathrm{B}$. $^{21}$

Several studies have indicated a role for ceramide in various types of apoptosis through the activation of aSMase, leading to the modulation of BAD/BAX, Apaf1, cytochrome $c$ and the activation of the SAPK/JNK pathway. ${ }^{13,22}$ On the other hand, SMases can also be activated by positive signals elicited by PDGF, CD28 and oxidized LDL resulting in the transcription of growth-related genes through the activation of the MAPK pathway and upregulation of a plethora of essential cellular functions like cell growth, differentiation and repair. ${ }^{23-25}$ In the negative signaling pathway, ceramide behaves as a second messenger in the initiation of apoptotic cell death in a variety of pathophysiologic settings. ${ }^{26}$ Application of exogenous ceramide alone, using cell-permeable ceramide analogues such as $\mathrm{C}_{2}, \mathrm{C}_{6}$ and $\mathrm{C}_{8}$ isoforms, is sufficient to initiate apoptosis in some cells. ${ }^{27}$ In contrast, the use of fumonisin B1, a specific ceramide synthase inhibitor (Figure 1), provides marked protection against chemical hypoxia-induced DNA strand breaks, DNA fragmentation and cell death. ${ }^{28}$ Recent investigations have shown that in some experimental systems, CD95 signals via ceramide-rich membrane rafts, suggesting that ceramide might participate in the clustering of CD95. ${ }^{29,30}$

Although many studies have shown the role of ceramide in apoptosis, most of these studies were performed in cell-free systems with analogues of ceramide. In addition, the effect of ceramide has been shown to vary from system to system, with results ranging from regulating apoptosis to promoting mitogenic responses. We have evaluated the importance of ceramide and other sphingolipid intermediates in activationinduced apoptosis, and demonstrated that de novo synthesis of the sphingolipid intermediate, sphinganine, but not ceramide, is important for TCR-signaled cell death following activation. The effect of these intermediates seems to be exerted through modulating the early steps of CD95-mediated caspase activation. This is the first time that sphingolipid intermediates are being shown to be important in the CD95 signaling process.

\section{Results}

\section{Myriocin inhibits activation-induced apoptosis}

Myriocin (ISP-1, thermozymocidin) is a newly identified immunosuppressant ${ }^{31}$ with a structure homologous to sphingosine. A natural product of Isaria sinclairii, myriocin specifically inhibits serine palmitoyl transferase, ${ }^{32}$ the first enzyme in the de novo synthesis of ceramide (Figure 1). Miyake et al. ${ }^{32}$ found that myriocin suppressed proliferation of an IL-2-dependent mouse cytotoxic T-cell line, CTLL-2, in a dose-dependent manner. Suppression of CTLL-2 proliferation with myriocin was found to be a result of the inhibition of serine palmitoyl transferase (Figure 1). The serine palmitoyl transferase enzyme consists of two subunits, LCB1/SPTLC1 and LCB2/SPTLC2. ${ }^{33}$ Myriocin inhibits serine palmitoyl transferase activity by binding directly to LCB1 and LCB2. ${ }^{34}$ Deoxomyriocin is a synthesized analog of myriocin found to be 5 to 10 -fold more potent than myriocin (data not shown). Since sphingolipids such as ceramide have been implied to play a critical role in the pathway leading to apoptosis, we first examined the effect of myriocin on AICD. T-cell hybridoma A1.1 cells were activated with anti-CD3 immobilized on tissue culture plastic plates overnight in the presence or absence of myriocin. We found that myriocin inhibited AICD in a concentration-dependent manner, with $50 \%$ inhibition at $3.125 \mu \mathrm{M}$ and complete inhibition at $6.25 \mu \mathrm{M}$. As shown in Figure $2 \mathrm{a}, \mathrm{A} 1.1$ cells activated with anti-CD3 show membrane blebbing, characteristic of cells undergoing apoptosis. On the other hand, A1.1 cells activated with anti-CD3 that were also treated with $6.25 \mu \mathrm{M}$ myriocin appeared round and healthy, illustrating that myriocin is effective in inhibiting AICD. At this concentration, myriocin did not have any 
a

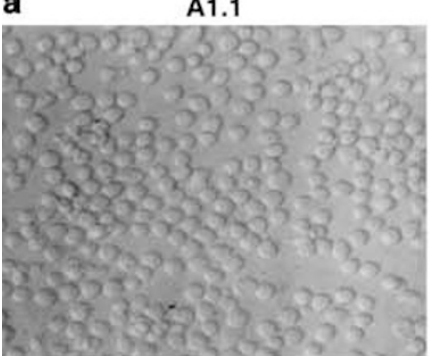

Myriocin

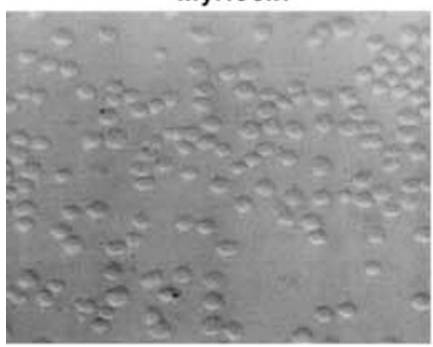

b
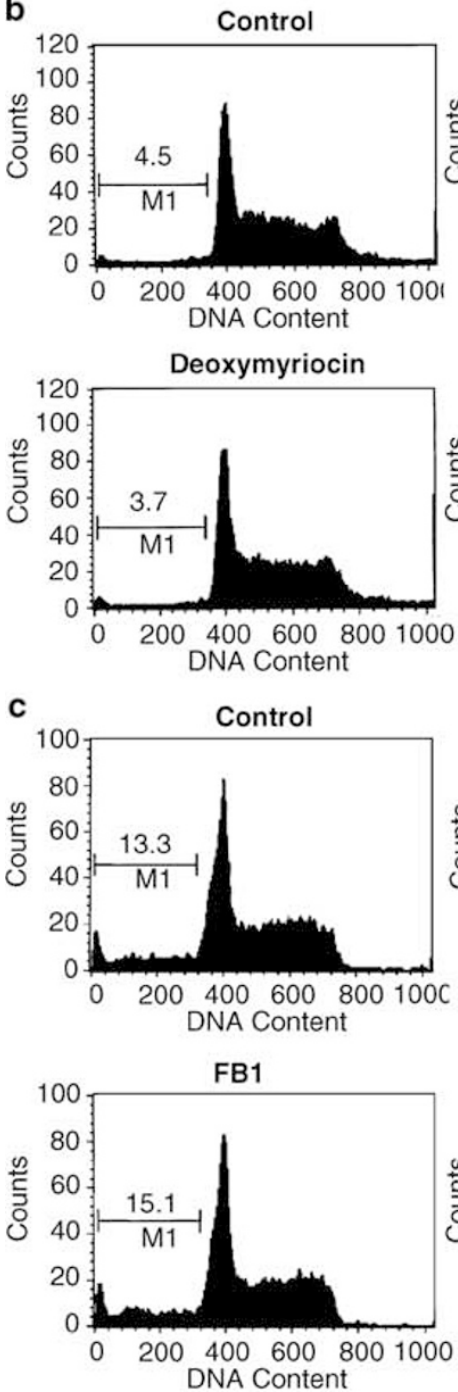

Anti-CD3

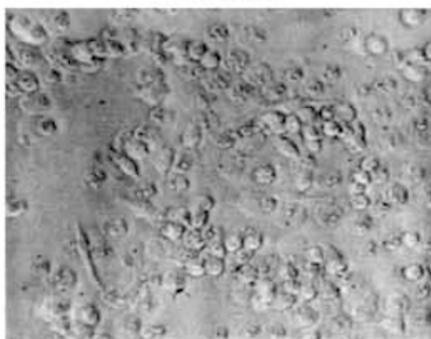

Anti-CD3 + Myriocin
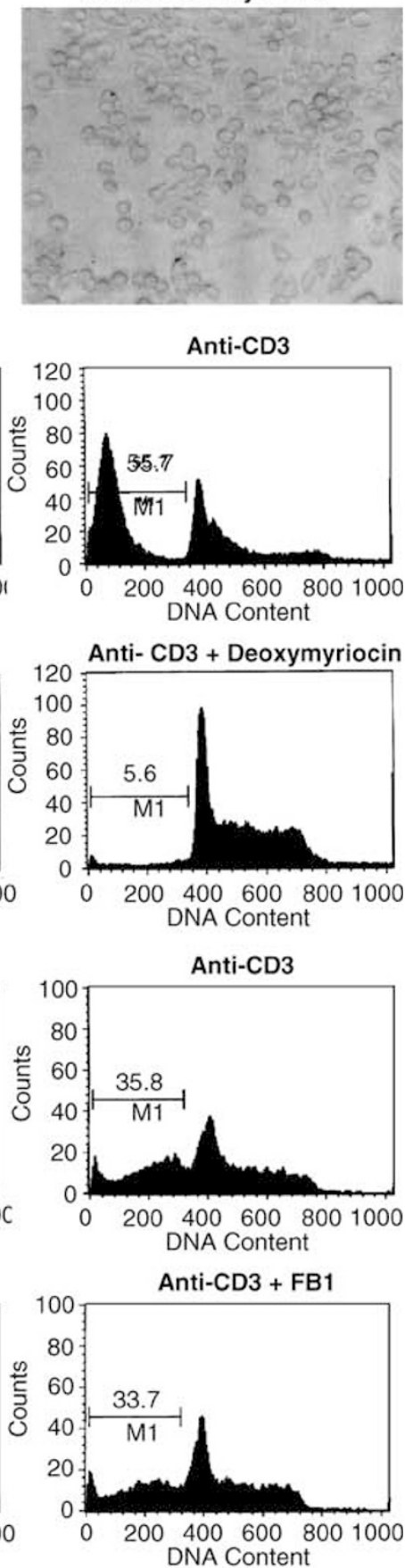

Figure 2 Myriocin inhibits AICD. (a) A1.1 cells were activated with plastic-bound anti-CD3 alone with or without $6.25 \mu \mathrm{M}$ myriocin. Photographs were taken $14 \mathrm{~h}$ after activation. (b) and (c) A1.1 cells were activated with anti-CD3 in the presence and absence of $6.25 \mu \mathrm{M}$ deoxomyriocin or fumonisin B1 for $14 \mathrm{~h}$. Apoptosis was determined by DNA content analysis. The marked region represents percent of apoptotic cells in the subdiploid peak 
effect on cell proliferation in unactivated cells (data not shown). We also looked at whether deoxomyriocin could inhibit AICD. Anti-CD3 treatment induced apoptosis in A1.1 cells as demonstrated by the presence of a hypodiploid peak in DNA content analysis (Figure 2b). Anti-CD3-treated A1.1 cells also treated with deoxomyriocin did not have a hypodiploid peak, indicating that deoxomyriocin can also inhibit apoptosis in A1.1 cells.

To reveal whether de novo synthesis of ceramide plays a role in the protection of cells from activation-induced apoptosis, we examined the effect of the ceramide synthase inhibitor, Fumonisin B1 (FB1), ${ }^{35}$ which inhibits the final step in ceramide synthesis. FB1 is the most common mycotoxin produced by Fusarium moniliforme. The structure of FB1 resembles sphingosine and studies indicate that FB1 inhibits sphingolipid biosynthesis by inhibiting ceramide synthase activity. FB1 interacts with the binding sites for sphinganine and fatty acyl-coenzyme $A(C o A)^{36}$ in a competitive manner, causing sphinganine to accumulate. Wang et al. ${ }^{35}$ showed that the $\mathrm{IC}_{50}$ of $\mathrm{FB} 1$ was approximately $0.1 \mu \mathrm{M}$ for inhibiting the incorporation of serine into sphingosine. Anti-CD3 treatment induced apoptosis in A1.1 cells as demonstrated by the hypodiploid peak in DNA content analysis (Figure 2c). Activated A1.1 cells treated with FB1 also had a hypodiploid peak. Therefore, blockade of ceramide synthesis using FB1 does not prevent AICD. We also found that ceramide analogues $\mathrm{C} 2$ and $\mathrm{C} 6$ could not induce apoptosis in $\mathrm{A} 1.1$ cells, nor could these ceramide analogues reverse the inhibitory effect of myriocin upon anti-CD3 activation (data not shown). These results reveal that although sphingolipid synthesis is required as indicated by the capability of myriocin to inhibit AICD, de novo synthesis of ceramide is not necessary for activation-induced apoptosis.

To test the effect of myriocin on activation-induced apoptosis in other cells, we employed another T-cell hybridoma IE5. As in A1.1 cells, AICD in IE5 cells is also dependent on Fas and FasL interaction (data not shown). As shown in Figure $3 a$, anti-CD3-induced AICD in these cells is blocked by myriocin. In addition, we also generated T-cell blasts from freshly isolated splenocytes and tested for AICD according to the established protocol. ${ }^{3,4}$ We found that AICD in T-cell blasts was also inhibited by myriocin (Figure $3 b$ ). Furthermore, we have found that apoptosis in A1.1 cells induced by dexamethasone and cisplatin is not affected by myriocin (data not shown), indicating the specificity of myriocin.

\section{Sphinganine reverses the effect of myriocin}

The synthesis of sphingolipids is a multistep process. Sphinganine is an intermediate upstream of ceramide synthase, an enzyme sensitive to FB1. If this intermediate participates in Fas-mediated apoptosis, it should be able to reverse the inhibitory effect of myriocin. To test this hypothesis, we activated A1.1 cells with anti-CD3 in the presence of both myriocin and sphinganine, and assessed apoptosis by DNA content analysis. As shown above, myriocin can inhibit AICD, as seen by the absence of the subdiploid peak. Interestingly, when cells were treated with both myriocin and sphinganine, a subdiploid peak reappeared (Figure 4), a
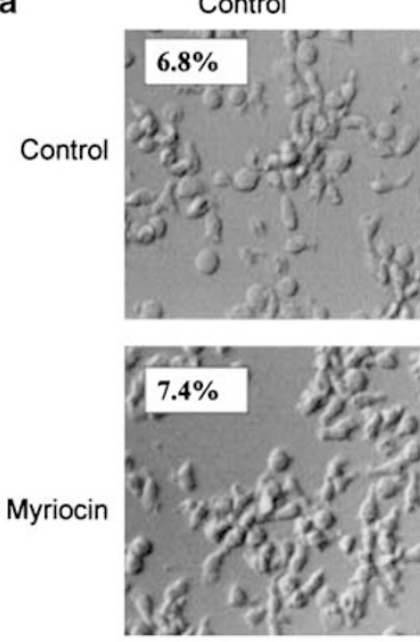

b
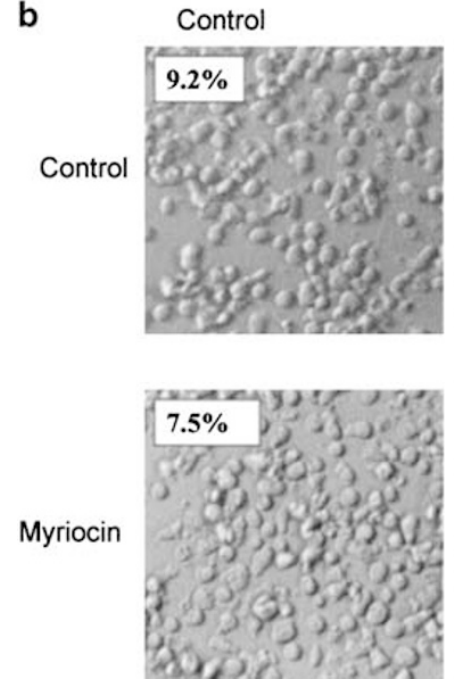

Anti-CD3
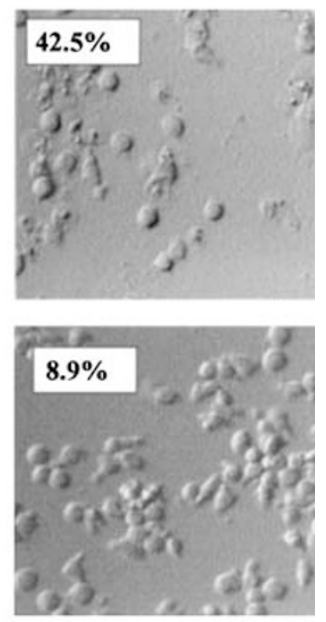

Anti-CD3
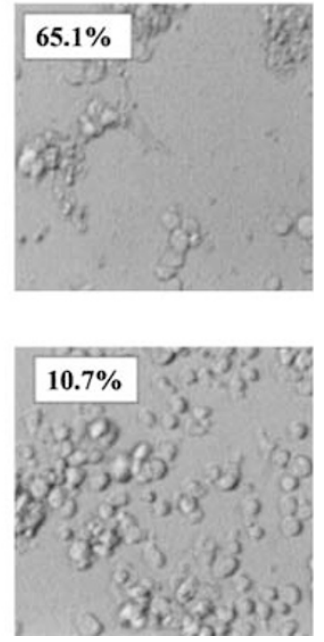

Figure 3 Myriocin inhibits AICD in other cells. T-cell hybridoma IE5 cells (a) and $T$ cell blasts (b) were activated with plastic-bound anti-CD3 for $12 \mathrm{~h}$ in the presence or absence of $6.25 \mu \mathrm{M}$ myriocin. Photographs represent cellular morphology, and percentage on the top left corners shows the ratio of apoptotic cells as determined by DNA content analysis

indicating that cells underwent apoptosis. This suggests that sphinganine is an important intermediate of the sphingolipid synthesis pathway participating in Fas-mediated apoptosis.

\section{Myriocin does not inhibit IL-2 production}

Activation of $\mathrm{A} 1.1 \mathrm{~T}$-cell hybridoma through the $\mathrm{T}$-cell receptor by antigen plus $\mathrm{MHC}$ or with anti-CD3 leads to apoptosis. ${ }^{37}$ Activation of T-cell hybridomas also leads to the production of cytokines such as IL-2. ${ }^{38}$ In order to determine whether myriocin inhibits AICD by interfering with TCR signaling, we examined if myriocin had an effect on IL-2 production. A1.1 cells were treated with myriocin, $\mathrm{C} 2$ or $\mathrm{C} 6$ ceramides in the presence or absence of anti-CD3 for $14 \mathrm{~h}$. Culture supernatants were examined for IL-2 production by ELISA. As shown in Figure 5, unactivated A1.1 cells do not produce detectable amounts of IL-2, while anti-CD3 activation leads to 

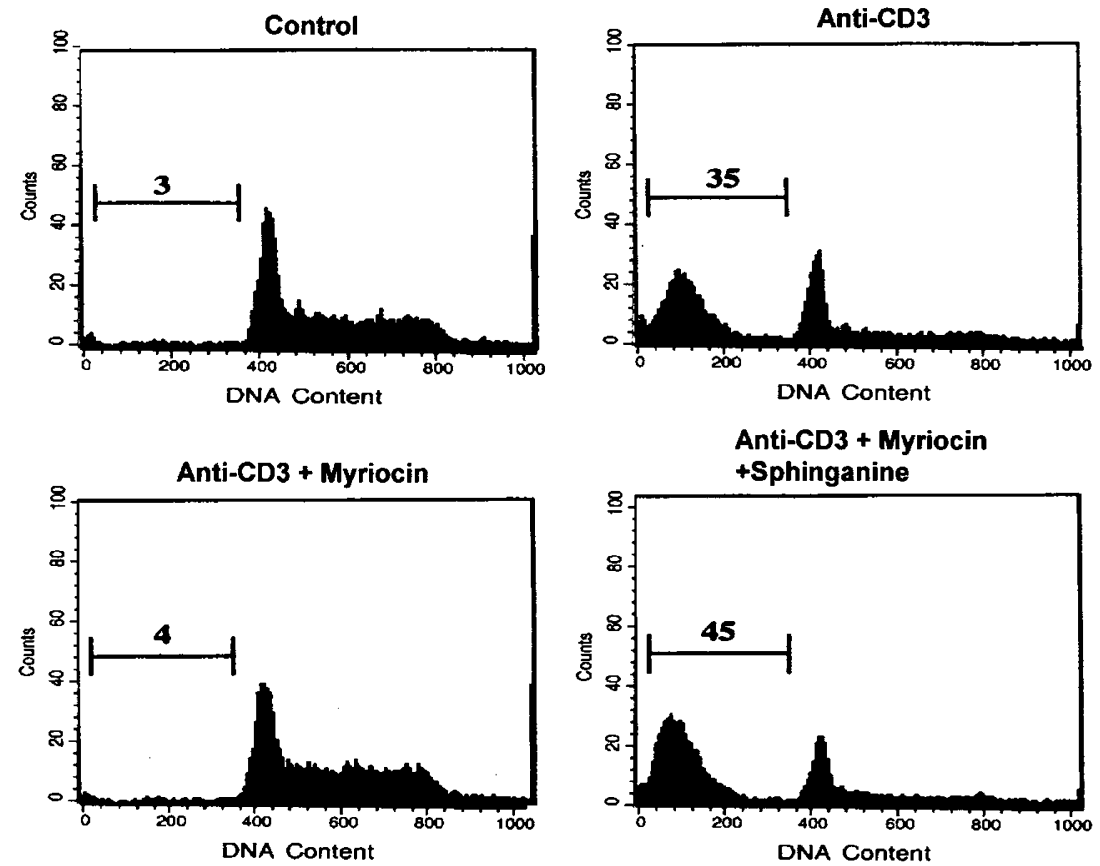

Figure 4 Sphinganine reverses the effect of myriocin. A1.1 cells were activated with anti-CD3 in the presence and absence of $6.25 \mu \mathrm{M}$ deoxomyriocin and $3.13 \mu \mathrm{M}$ sphinganine for $14 \mathrm{~h}$. Apoptosis was determined by DNA content analysis. The marked region represents percent of apoptotic cells in the subdiploid peak

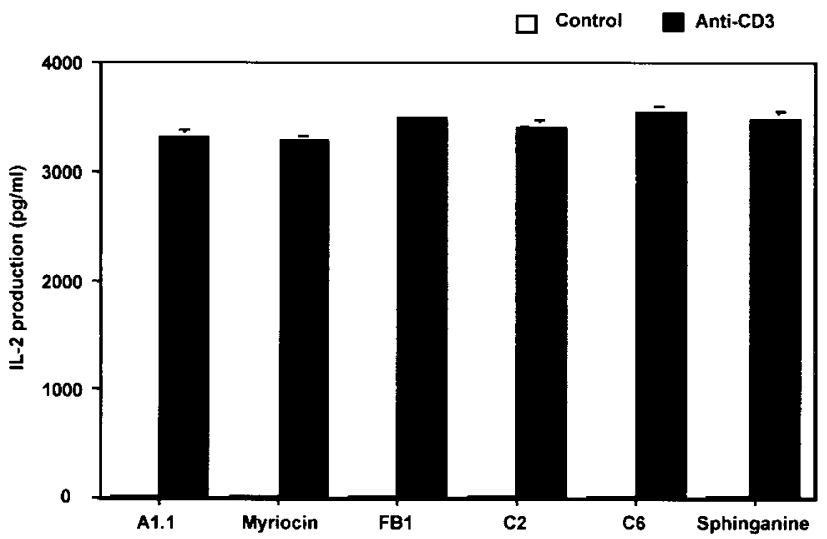

Figure 5 Myriocin does not inhibit activation-induced IL-2 production. A1.1 cells were treated with $6.25 \mu \mathrm{M}$ myriocin, $\mathrm{FB} 1$, sphinganine, $\mathrm{C} 2$ or $\mathrm{C} 6$ ceramide in the presence or absence of anti-CD3 for $14 \mathrm{~h}$. Culture supernatants were examined for IL-2 production by ELISA

a significant increase in IL-2 production. Cells treated with anti-CD3 as well as myriocin also produced significant amounts of IL-2, illustrating that TCR signaling is not compromised and that myriocin inhibits apoptosis downstream of TCR signaling. Similarly, FB1, C2, C6 and sphinganine also did not affect IL-2 production. Therefore, sphingolipids are not involved in general TCR activation process.

\section{Myriocin does not affect CD95 or CD95L expression}

Crosslinking the TCR of T-cell hybridomas leads to AICD. AICD in T-cell hybridomas have been shown to require the

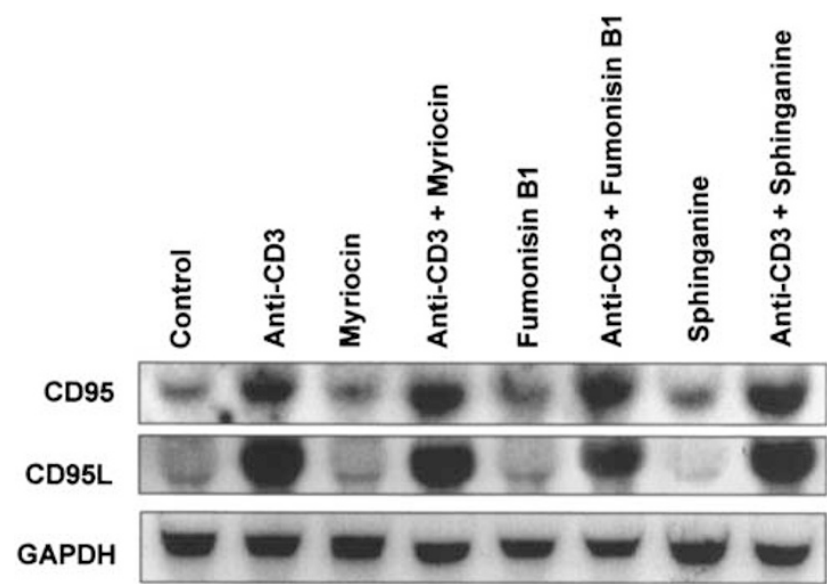

Figure 6 Myriocin does not affect CD95 and CD95L expression. A1.1 cells were treated with or without anti-CD3 in the presence or absence of $6.25 \mu \mathrm{M}$ myriocin, fumonisin $\mathrm{B} 1$ or sphinganine for $4 \mathrm{~h}$. Total RNA was isolated for Northern blot analysis for the expression of CD95, CD95L and GAPDH

expression of CD95 and CD95L. ${ }^{39}$ We tested whether the inhibitory effect of myriocin on AICD is because of its effect on activation-induced CD95 and CD95L expression. A1.1 cells express low basal levels of CD95 that increase within 2-4h upon activation with anti-CD3. On the other hand, A1.1 cells do not express CD95L unless they are activated with antiCD3. ${ }^{40}$ CD95L expression can be seen in A1.1 cells at 4-6h after activation with anti-CD3. ${ }^{41}$ We found that neither myriocin nor FB1 affects CD95 or CD95L expression upon activation with ani-CD3 (Figure 6). Therefore, the effect of sphingolipids is not exerted by interfering with activationinduced CD95 and CD95L expression. 


\section{Myriocin directly inhibits CD95 signaling}

Since myriocin does not affect CD95 and CD95L expression, its effect is likely through the death signal after Fas stimulation. Therefore, we tested the effect of myriocin on CD95-mediated cell death signaling. DNA content analysis of A1.1 cells shows that few cells are in the subdiploid peak (Figure 7a). Treatment with anti-CD3 dramatically increased the number of cells in the subdiploid peak. As demonstrated previously, ${ }^{2}$ cells treated with both anti-CD3 and CsA are inhibited from undergoing apoptosis, illustrated by the diminished subdiploid peak. CsA inhibits FasL expression, thereby inhibiting apoptosis. ${ }^{42}$ However, cells rescued by CsA are still sensitive to Fas ligation with $\mathrm{JO} 2 . \mathrm{JO} 2$ is an antimouse Fas monoclonal antibody with activation capacity. Interestingly, cells rescued with CsA and further treated with $\mathrm{JO} 2$ are still inhibited from undergoing apoptosis by myriocin, indicating that the effect of myriocin is downstream of the Fas death signal. In order to further support that myriocin affects CD95-mediated death pathway, we utilized A20 B cells. A20 is a murine B-cell line that expresses CD95 and is very sensitive to CD95 ligation-induced apoptosis. Cells were incubated with $\mathrm{JO} 2$ in the presence or absence of myriocin. We found that $\mathrm{JO} 2$-induced apoptosis also was inhibited by myriocin in A20 B cells (Figure 7b). Since myriocin does not modulate the transcriptional regulation of CD95 or CD95L, this result suggests that the inhibitory effect of myriocin is specifically involved in the CD95-death pathway.

\section{Myriocin inhibits caspases 3, 8 and 9 activation}

CD95 is a cell surface death receptor that belongs to the tumor necrosis factor receptor superfamily. Upon binding of CD95 to CD95L, CD95 trimerizes and brings together three intracellular death domains that bind to the death domain of FADD. FADD also contains a death effector domain (DED) that recruits the DED of procaspase 8 to form the death-inducing signaling complex (DISC). When procaspase 8 is bound to the DISC, it is cleaved in order to yield active caspase 8 , thereby initiating the caspase cascade. ${ }^{43}$ Since myriocin did not affect CD95 or CD95L expression, we examined whether it affects the death signaling pathway. Figure 8 illustrates that $A 1.1$
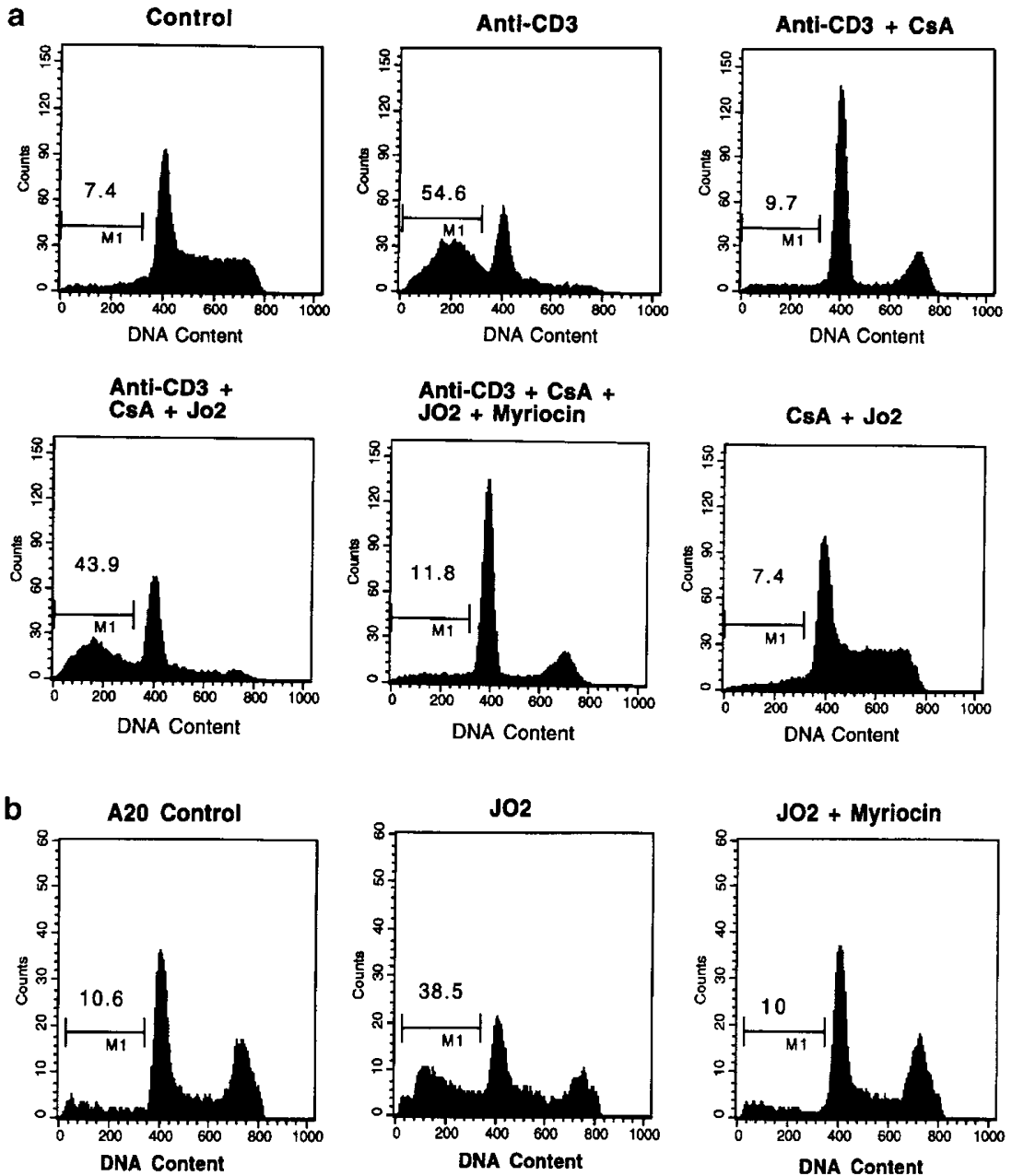

Figure 7 Myriocin directly inhibits CD95 signaling. (a) A1.1 cells were treated with anti-CD3, $200 \mathrm{ng} / \mathrm{ml}$ cyclosporin A, $200 \mathrm{ng} / \mathrm{ml}$ JO2 in the presence or absence of $6 \mu \mathrm{M}$ myriocin for $12 \mathrm{~h}$. Apoptosis was determined by DNA content analysis. The marked region represents percent of apoptotic cells in the subdiploid peak. (b) A20 cells were treated with $10 \mathrm{ng} / \mathrm{ml} \mathrm{JO2}$ in the presence or absence of $6 \mu \mathrm{M}$ myriocin for $12 \mathrm{~h}$. Apoptosis was determined by DNA content analysis 

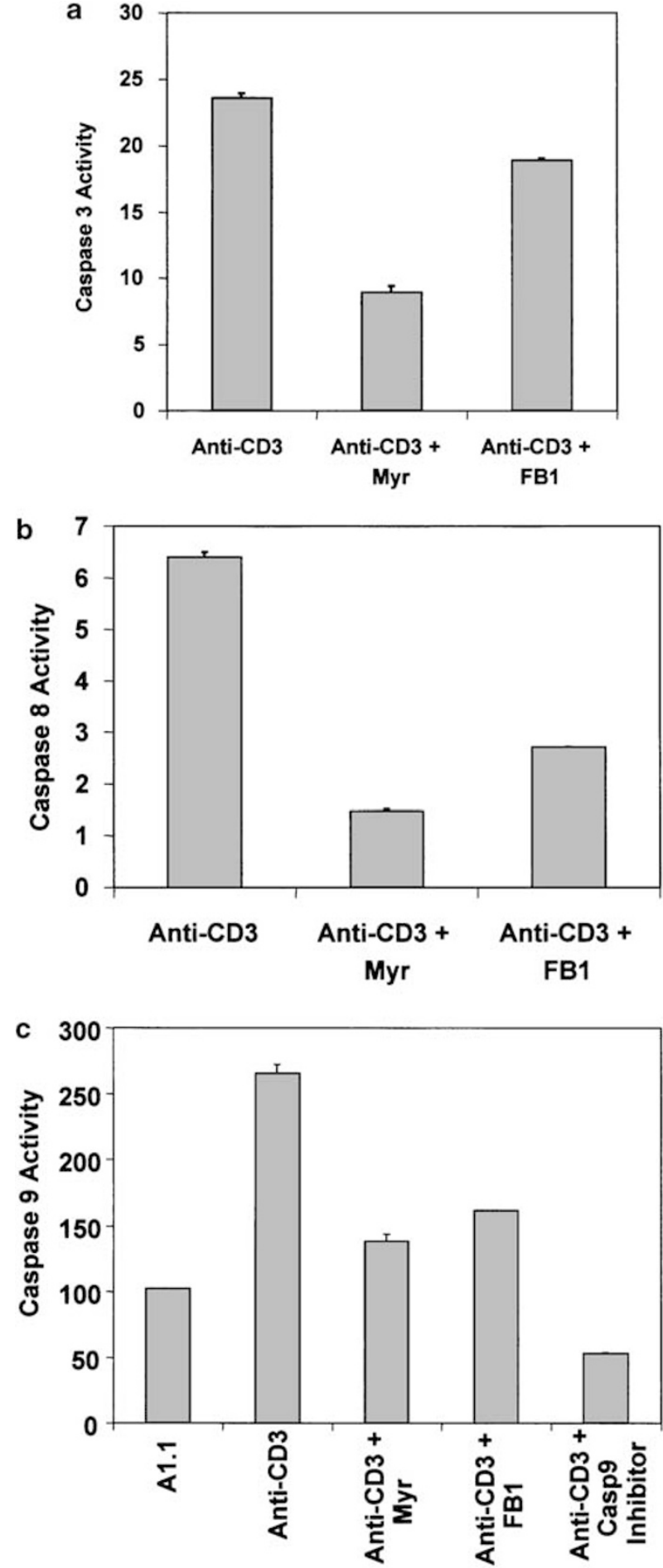

Figure 8 Myriocin inhibits caspases $-3,-8$ and -9 activation. A1.1 cells were activated with anti-CD3 and treated with $12.5 \mu \mathrm{M}$ myriocin for $5 \mathrm{~h}$. Cells were harvested, lysed and analyzed for caspase activity using Apoalert Caspase-3 (a), caspase-8 (b) and caspase-9 (c) fluorescent assay kit. Fluorescence was determined at $400 \mathrm{~nm}$ excitation and $505 \mathrm{~nm}$ emission

cells treated with anti-CD3 have high levels of caspases 3 (Figure 8a), 8 (Figure 8b) and 9 (Figure 8c) activity. The activation of these caspases was inhibited by myriocin. Since both caspases 8 and 9 , two initiators of the caspase cascade, were inhibited, it is likely that the effect of myriocin is exerted through modulating the DISC formation process.

\section{Discussion}

Sphingolipids are key components of the lipid bilayer that can also serve as vital second messengers in cell survival, proliferation, cell-cell communication, cytokine production and apoptosis. ${ }^{44-46}$ The role of sphingolipids in T lymphocytes following TCR activation is beginning to be identified. For example, a recent study has revealed that membrane rafts play a critical role in concentrating $\mathrm{MHC}$ class II molecules into microdomains that allow efficient antigen presentation at low ligand densities. ${ }^{47}$ The importance of modular interaction during transmembrane signaling is rapidly being realized. These membrane microdomains or rafts serve as privileged sites where receptors and proximal signaling molecules optimally interact. ${ }^{48}$ However, the role of membrane rafts in AICD is currently not known. In this study, we addressed the possible involvement of sphingolipids in the regulation of AICD. We utilized two enzyme inhibitors and determined whether it is ceramide or another sphingolipid intermediate that is important for AICD in A1.1 T cells. A1.1 cells were analyzed following activation with anti-CD3 and treatments with sphingolipid intermediates or sphingolipid synthesis inhibitors, and found an important role of the sphingolipid synthesis intermediates in the modulation of the CD95 death pathway. Our results indicate that the effect of the sphingolipid intermediates is associated with the early steps of CD95mediated caspase activation, likely by participating in DISC formation.

Sphingolipid synthesis starts with the condensation of palmitoyl CoA with serine to form 3-ketosphinganine, a process catalyzed by serine palmitoyl transferase. 3-Ketosphinganine is subsequently reduced to form sphinganine, then acylated by ceramide synthase to yield dihydroceramide, which is further oxidized to yield ceramide, where a trans- 4,5 double bond is introduced by the enzyme dihydroceramide reductase. ${ }^{13}$ In this study, we employed a serine palmitoylCoA transferase inhibitor, myriocin, to block sphingolipid synthesis. This inhibitor has been shown to be highly effective and specific in inhibiting the activity of serine-palmitoyl transferase by binding to the enzyme. ${ }^{32,34}$ As previously mentioned, serine-palmitoyl transferase consists of two subunits: LCB1 and LCB2. Myriocin specifically inhibits serine-palmitoyl transferase activity by binding to LCB1 and LCB2. ${ }^{34}$ We have discovered that inhibition of serinepalmitoyl transferase with myriocin, but not of ceramide synthase with FB1, could abrogate CD95-mediated apoptosis. Since myriocin was able to inhibit apoptosis and FB1 was not, it is conceivable that a sphingolipid intermediate upstream of ceramide synthase and downstream of serine-palmitoyl transferase, rather than ceramide itself, is critical for CD95induced activation of the caspase cascade. Consistent with this notion, we found that addition of sphinganine, but not ceramide analogues, could reverse the inhibitory effect of myriocin. 
Initial studies on the effects of myriocin revealed that it inhibits both CTLL-2 cell proliferation and mouse allogeneic mixed lymphocyte reaction by inhibiting serine-palmitoyl transferase. ${ }^{31,32}$ It was later discovered that the decrease in CTLL-2 cell number upon treatment with myriocin was not because of the inhibition of cell cycle progression but a consequence of apoptosis. ${ }^{49}$ However, the same group found that myriocin did not induce apoptosis in a mouse pro-B cell line, indicating that the effect of myriocin depends on the cell type. Our experiments show that myriocin does not affect IL-2 production (Figure 5) and is similar to the results from Fujita et al. $^{31}$ Inhibition of apoptosis by myriocin is not limited to A1.1 cells or to CD95-mediated apoptosis. As we have shown, myriocin also inhibits apoptosis in A20 B cells (Figure 7b), IE5 T-cell hybridoma (Figure $3 \mathrm{a}$ ) and freshly generated T-cell blasts (Figure 3b). However, myriocin does not seem to affect dexamethasone-induced apoptosis of thymocytes (data not shown). The various effects of myriocin are probably owing to the different amounts and type of sphingolipids expressed in different cell types. Ratios of different sphingolipid intermediates might determine what effect myriocin will have on a specific cell type.

Toxicological studies with FB1 reveal that exposure to this toxin leads to an accumulation of high concentrations of the cytotoxic sphingolipid sphinganine. ${ }^{50,51}$ By inhibiting ceramide synthesis with FB1, sphinganine is prevented from being converted to ceramide, thus, sphinganine accumulates and facilitates cell death. It is worthy of note that although we did not see an inhibitory effect of FB1 on activation-induced apoptosis, we found that FB1 could inhibit radiation-induced apoptosis in A1.1 cells (data not shown). Therefore, the amount of FB1 we used is effective in modulating ceramide production and the effect we saw on AICD is not because of a general toxicity.

Our investigation has excluded the possibility that myriocin inhibits AICD by modulating CD95 and CD95L expression. We could not determine whether CD95 or CD95L surface expression was affected by myriocin because available antibodies do not stain FasL on A1.1 cells. However, in other T-cell hybridoma IE5, FasL could be detected by surface staining. We have found that activation-induced CD95 and CD95L expression on IE5 surface is not affected by myriocin (data not shown). Using Northern blot analysis, we discovered that CD95 and CD95L expressions were not affected by myriocin; therefore, we examined the effect of myriocin on CD95-induced cell death process. The ability of myriocin to prevent CD95-triggered apoptosis was supported by data from A20 cells. A20 cells treated with $\mathrm{JO} 2$ undergo CD95-mediated apoptosis. However, A20 cells treated with both $\mathrm{JO} 2$ and myriocin did not undergo apoptosis. This also suggests that myriocin inhibits the cell death signal following CD95 ligation. Since myriocin seemed to be inhibiting apoptosis following CD95 ligation, we examined whether caspase activation was affected. We found that myriocin is able to inhibit caspases 3, 8 and 9 activation. These results indicate modulation of caspases by sphingolipids during AICD, pointing to a novel regulatory mechanism. Thus, our studies reveal the requirement of de novo sphingolipid synthesis in AICD. Exogenous administration of sphinganine has been shown to induce apoptosis in hepatoma cells, a mechanism which involves caspase activation. ${ }^{52}$ Furthermore, CD95-mediated cell death has been shown previously to involve sphingolipids, ${ }^{53-55}$ but in a ceramide-independent mechanism. ${ }^{56,57}$ The precise mechanism of sphinganinemodulated caspase activation is not known; however, it may be involved with the formation of the DISC, since caspase 8 activation requires its recruitment into the DISC. Therefore, our observation that caspase activation is inhibited by myriocin may be because of the lack of DISC formation. We are currently determining whether myriocin can inhibit DISC formation and investigating whether raft formation is required.

\section{Materials and Methods}

\section{Cells and reagents}

A1. 1 murine T-cell hybridoma cells were maintained in RPMI-1640 media (GIBCO, Gaithersburg, MD, USA) with 5\% FBS (GIBCO, Gaithersburg, $M D, U S A)$ and full complement of antibiotics/antimycotics in humidified atmosphere supplemented with $5 \% \quad \mathrm{CO}_{2}$ at $37^{\circ} \mathrm{C}$. A20 cells were maintained as described by Mueller and Scott. ${ }^{58}$ Myriocin was obtained from Dr. Fujita. Fumonisin B1 (FB1), C2 Ceramide and C6 Ceramide, were purchased from Calbiochem (La Jolla, CA, USA). Sphinganine (DLerythro-dihydro-sphingosine) was purchased from Sigma (St. Louis, MO, USA).

\section{Generation of T-cell blasts}

Murine splenocytes were isolated from 10-week-old female Balb/c mice (National Cancer Institute, Frederick, MD, USA). Single-cell suspension was generated from spleen by pressing between the frosted ends of microscope slides and passing through $70 \mu \mathrm{m}$ nylon cell strainers. Cells were activated with anti-CD3 for $48 \mathrm{~h}$ and then maintained in the presence of IL-2 for another $48 \mathrm{~h}$. Cells were maintained in RPMI 1640 medium (Gibco/BRL, Gaithersburg, MD, USA), supplemented with $2 \mathrm{mM} \mathrm{L-}$ glutamine, $50 \mathrm{mM}$ 2-mercaptoethanol, 10\% heat-inactivated fetal bovine serum (Sigma, St. Louis, MO, USA), and $10 \mathrm{mM}$ gentamycin.

\section{DNA content analysis}

After treatment, cells were fixed with $70 \%$ ethanol for at least $30 \mathrm{~min}$ at $4{ }^{\circ} \mathrm{C}$, followed by two washes with PBS. The fixed splenocytes were then incubated in PBS containing propidium iodide (Sigma, St. Louis, MO, USA) at $50 \mu \mathrm{g} / \mathrm{ml}$ and RNase (Boehringer Mannheim, Indianapolis, IN, USA) at $0.1 \mathrm{mg} / \mathrm{ml}$ at room temperature for $30 \mathrm{~min}$. DNA content was determined by flow cytometry on FacScan (Becton Dickinson, San Jose, CA, USA). The FL2 intensity was plotted as histograms on a linear scale. Apoptotic cells were shown as a hypodiploid peak.

\section{Northern blotting}

Total RNA was isolated with affinity columns (QIAGEN, Chatsworth, CA, USA), according to the manufacturer's recommended protocol. RNA samples were fractionated on $1 \%$ agarose/2.2 $\mathrm{M}$ formaldehyde denaturing gel, and transferred onto a Nytran membrane (Schleicher \& Schuell, Inc., Keene, NH, USA). Mouse CD95 and CD95L cDNA probes (from Dr. Shigekazu Nagata, Osaka Bioscience Institute, Japan) were randomly primed with $\left[{ }^{32} \mathrm{P}\right]$-dCTP (Boehringer Mannheim, Indianapolis, IN, USA) according to the manufacturer's instructions. Prehybridization and hybridization were carried out at $42^{\circ} \mathrm{C}$ in a solution containing $5 \times \mathrm{SSC}$ 
( $10 \times \mathrm{SSC}$ is $1.5 \mathrm{M} \mathrm{NaCl}, 0.15 \mathrm{M}$ sodium citrate), $2.5 \mathrm{mM}$ EDTA, $0.1 \%$ SDS, $5 \times$ Denhardt's solution, $2 \mathrm{mM}$ sodium pyrophosphate, $50 \mathrm{mM}$ sodium phosphate, and $50 \%$ formamide. Membranes were washed with $0.2 \times \mathrm{SSC}, 0.1 \%$ SDS at $56^{\circ} \mathrm{C}$ for $1 \mathrm{~h}$ and hybridization signals were detected by autoradiography

\section{IL-2 ELISA}

IL-2 in culture supernatants was detected with the cytoscreen Immunoassay kit (Biosource, Camarillo, CA, USA) according to the manufacturer's recommended protocol. Recombinant murine IL-2 was used as standard. Briefly, the tissue culture supernatant was diluted in a standard diluent buffer included in the kit. Standard cytokines and samples were incubated on the first respective cytokine-specific antibodies-coated microtiter plates for $1.5 \mathrm{~h}$ at $37^{\circ} \mathrm{C}$. After washing, the bound cytokines were detected with biotinylated second cytokine-specific antibody and streptavidin peroxidase. The amount of cytokines was determined by the addition of tetramethyl benzidine. The amount of IL-2 was determined by recession curves obtained from recombinant murine IL-2 provided in the kit.

\section{Caspase assay}

Caspases-8, -9 and -3 activities were determined using ApoAlert Caspase Fluorescent Assay Kits (Clontech, Palo Alto, CA, USA) according to the manufacturer's recommended protocol. Briefly, upon treatments, $2 \times 10^{6}$ cells were resuspended in $50 \mu \mathrm{l}$ of lysis buffer included in the kits and incubated on ice for $10 \mathrm{~min}$. Upon centrifugation, the lysate supernatant was isolated and mixed with a $2 \times$ reaction buffer/DTT mix. Upon addition of the specific substrate peptide, labeled with chromophore $p$-nitroaniline, the reaction was carried out at $37^{\circ} \mathrm{C}$ for $1 \mathrm{~h}$. The activity of caspases was determined at $400 \mathrm{~nm}$ excitation and $505 \mathrm{~nm}$ emission.

\section{Acknowledgments}

We thank Dr. Liying Zhang, Miss Jennifer Hinshaw, Dr. Xiaoren Zhang, Dr. Ruoxiang Wang, Dr. Achsah Keegan, Dr. David Scott and Dr. Stephan Ladisch for their discussions and technical help. This work was supported by National Institutes of Health Grants R01Al43384, R01Al50222 and R01CA76492 to YFS and R01CA76492-03SI to JCS. KS is a recipient of Fonds de la recherche en Sante du Quebec postdoctoral fellowship. This work will be submitted in partial fulfillment of the requirements for the Doctor of Philosophy degree (J.C.S.), Molecular and Cellular Oncology Program, Institute for Biomedical Sciences of the Columbian School of Arts and Sciences, The George Washington University.

\section{References}

1. Mercep M, Noguchi PD and Ashwell JD (1989) The cell cycle block and lysis of an activated $\mathrm{T}$ cell hybridoma are distinct processes with different $\mathrm{Ca}^{2+}$ requirements and sensitivity to cyclosporine A. J. Immunol. 142: 4085-4092

2. Shi YF, Sahai BM and Green DR (1989) Cyclosporin A inhibits activationinduced cell death in T-cell hybridomas and thymocytes. Nature 339: 625-626

3. Lenardo MJ (1991) Interleukin-2 programs mouse alpha beta T lymphocytes for apoptosis. Nature 353: 858-861

4. Radvanyi LG, Mills GB and Miller RG (1993) Religation of the T cell receptor after primary activation of mature $\mathrm{T}$ cells inhibits proliferation and induces apoptotic cell death. J. Immunol. 150: 5704-5715

5. Russell JH (1995) Activation-induced death of mature T cells in the regulation of immune responses. Curr. Opin. Immunol. 7: 382-388
6. Scott DW, Grdina T and Shi Y (1996) T cells commit suicide, but B cells are murdered! J. Immunol. 156: 2352-2356

7. Sharma K, Wang RX, Zhang LY, Yin DL, Luo XY, Solomon JC, Jiang RF, Markos K, Davidson W, Scott DW et al. (2000) Death the Fas way: regulation and pathophysiology of CD95 and its ligand. Pharmacol. Ther. 88: 333-347

8. Nagata S and Suda T (1995) Fas and Fas ligand: Ipr and gld mutations. Immunol. Today 16: 39-43

9. Cohen PL and Eisenberg RA (1991) Lpr and gld: single gene models of systemic autoimmunity and lymphoproliferative disease. Annu. Rev. Immunol. 9: 243-269

10. Nagata S and Golstein P (1995) The Fas death factor. Science 267: 14491456

11. Bratton SB, MacFarlane M, Cain K and Cohen GM (2000) Protein complexes activate distinct caspase cascades in death receptor and stress-induced apoptosis. Exp. Cell Res. 256: 27-33.

12. Tang D and Kidd VJ (1998) Cleavage of DFF-45/ICAD by multiple caspases is essential for its function during apoptosis. J. Biol. Chem. 273: 28549-28552

13. Bose R, Verheii M, Haimovitz-Friedman A, Scotto K, Fuks Z and Kolesnick R (1995) Ceramide synthase mediates daunorubicin-induced apoptosis: an alternative mechanism for generating death signals. Cell 82: 405-414

14. Okazaki T, Bielawska A, Domae N, Bell RM and Hannun YA (1994) Characteristics and partial purification of a novel cytosolic, magnesiumindependent, neutral sphingomyelinase activated in the early signal transduction of 1 alpha,25-dihydroxyvitamin D3-induced $\mathrm{HL}-60$ cell differentiation. J. Biol. Chem. 269: 4070-4077

15. Spence MW, Wakkary J, Clarke JT and Cook HW (1982) Localization of neutral magnesium-stimulated sphingomyelinase in plasma membrane of cultured neuroblastoma cells. Biochim. Biophys. Acta 719: 162-164

16. Hannun YA and Obeid LM (1995) Ceramide: an intracellular signal for apoptosis. Trends Biochem. Sci. 20: 73-77

17. Jayadev S, Liu B, Bielawska AE, Lee JY, Nazaire F, Pushkareva M, Obeid LM and Hannun YA (1995) Role for ceramide in cell cycle arrest. J. Biol. Chem. 270: 2047-2052.

18. Pena LA, Fuks $Z$ and Kolesnick R (1997) Stress-induced apoptosis and the sphingomyelin pathway. Biochem. Pharmacol. 53: 615-621.

19. Ping SE and Barrett GL (1998) Ceramide can induce cell death in sensory neurons, whereas ceramide analogues and sphingosine promote survival. J. Neurosci. Res. 54: 206-213

20. Olivera A, Buckley NE and Spiegel S (1992) Sphingomyelinase and cellpermeable ceramide analogs stimulate cellular proliferation in quiescent Swiss 3T3 fibroblasts. J. Biol. Chem. 267: 26 121-26 127

21. Boland MP and O'Neill LA (1998) Ceramide activates NFkappaB by inducing the processing of p105. J. Biol. Chem. 273: 15 494-15500

22. Kolesnick RN and Kronke M (1998) Regulation of ceramide production and apoptosis. Annu. Rev. Physiol. 60: 643-665

23. Jarvis WD and Grant S (1998) The role of ceramide in the cellular response to cytotoxic agents. Curr. Opin. Oncol. 10: 552-559.

24. Spiegel S and Merrill Jr AH (1996) Sphingolipid metabolism and cell growth regulation. FASEB J. 10: 1388-1397

25. Spiegel S, Foster D and Kolesnick R (1996) Signal transduction through lipid second messengers. Curr. Opin. Cell Biol. 8: 159-167

26. Adam-Klages S, Schwandner R, Adam D, Kreder D, Bernardo K and Kronke M (1998) Distinct adapter proteins mediate acid versus neutral sphingomyelinase activation through the p55 receptor for tumor necrosis factor. J. Leukoc. Biol. 63: 678-682

27. Irie F and Hirabayashi Y (1998) Application of exogenous ceramide to cultured rat spinal motoneurons promotes survival or death by regulation of apoptosis depending on its concentrations. J. Neurosci. Res. 54: 475-485

28. Ueda N, Kaushal GP, Hong X and Shah SV (1998) Role of enhanced ceramide generation in DNA damage and cell death in chemical hypoxic injury to LLCPK1 cells. Kidney Int. 54: 399-406

29. Grassme H, Schwarz H and Gulbins E (2001) Molecular mechanisms of ceramide-mediated CD95 clustering. Biochem. Biophys. Res. Commun. 284: $1016-1030$

30. Grassme H, Jekle A, Riehle A, Schwarz H, Berger J, Sandhoff K, Kolesnick R and Gulbins $E$ (2001) CD95 signaling via ceramide-rich membrane rafts. J. Biol. Chem. 276: 20 589-20596

31. Fujita T, Inoue K, Yamamoto S, Ikumoto T, Sasaki S, Toyama R, Chiba K, Hoshino $Y$ and Okumoto T (1994) Fungal metabolites. Part 11. A potent 
immunosuppressive activity found in Isaria sinclairii metabolite. J. Antibiot. (Tokyo) 47: 208-215

32. Miyake Y, Kozutsumi Y, Nakamura S, Fujita T and Kawasaki T (1995) Serine palmitoyltransferase is the primary target of a sphingosine-like immunosuppressant, ISP-1/myriocin. Biochem. Biophys. Res. Commun. 211 396-403

33. Hanada K, Hara T and Nishijima M (2000) Purification of the serine palmitoyltransferase complex responsible for sphingoid base synthesis by using affinity peptide chromatography techniques. J. Biol. Chem. 275: 84098415

34. Chen JK, Lane WS and Schreiber SL (1999) The identification of myriocinbinding proteins. Chem. Biol. 6: 221-235

35. Wang E, Norred WP, Bacon CW, Riley RT and Merrill Jr AH (1991) Inhibition of sphingolipid biosynthesis by fumonisins. Implications for diseases associated with Fusarium moniliforme. J. Biol. Chem. 266: 14 486-14 490

36. Merrill Jr AH, van Echten G, Wang E and Sandhoff K (1993) Fumonisin B1 inhibits sphingosine (sphinganine) $\mathrm{N}$-acyltransferase and de novo sphingolipid biosynthesis in cultured neurons in situ. J. Biol. Chem. 268: 27 299-27 306

37. Shi YF, Szalay MG, Paskar L, Sahai BM, Boyer M, Singh B and Green DR (1990) Activation-induced cell death in T cell hybridomas is due to apoptosis. Morphologic aspects and DNA fragmentation. J. Immunol. 144: 3326-3333

38. Fotedar A, Boyer M, Smart W, Widtman J, Fraga E and Singh B (1985) Fine specificity of antigen recognition by $T$ cell hybridoma clones specific for poly-18: a synthetic polypeptide antigen of defined sequence and conformation. J. Immunol. 135: 3028-3033.

39. Brunner T, Mogil RJ, LaFace D, Yoo NJ, Mahboubi A, Echeverri F, Martin SJ, Force WR, Lynch DH, Ware CF et al. (1995) Cell-autonomous Fas (CD95)/Fasligand interaction mediates activation-induced apoptosis in T-cell hybridomas. Nature 373: 441-444

40. Wang R, Zhang L, Yin D, Mufson RA and Shi Y (1998). Protein kinase C regulates Fas (CD95/APO-1) expression. J. Immunol. 161: 2201-2207

41. Wang R, Zhang L, Zhang X, Moreno J, Luo X, Tondravi M and Shi Y (2001) Differential regulation of the expression of CD95 ligand, receptor activator of nuclear factor-kappa B ligand (RANKL), TNF-related apoptosis-inducing ligand (TRAIL), and TNF-alpha during T cell activation. J. Immunol. 166: 1983-1990

42. Brunner T, Yoo NJ, LaFace D, Ware CF and Green DR (1996) Activationinduced cell death in murine $\mathrm{T}$ cell hybridomas. Differential regulation of Fas (CD95) versus Fas ligand expression by cyclosporin A and FK506. Int. Immunol. 8: 1017-1026. abs.html

43. Budihardjo I, Oliver H, Lutter M, Luo X and Wang X (1999) Biochemical pathways of caspase activation during apoptosis. Annu. Rev. Cell Dev. Biol. 15: 269-290

44. Merrill Jr AH, Sullards MC, Wang E, Voss KA and Riley RT (2001) Sphingolipid metabolism: roles in signal transduction and disruption by fumonisins. Environ Health Perspect. 109(Suppl. 2): 283-289
45. Flores I, Jones DR and Merida I (2000) Changes in the balance between mitogenic and antimitogenic lipid second messengers during proliferation, cell arrest, and apoptosis in T-lymphocytes. FASEB J. 14: 1873-1875

46. Ballou LR (1992) Sphingolipids and cell function. Immunol. Today 13 339-341

47. Anderson HA, Hiltbold EM and Roche PA (2000) Concentration of MHC class II molecules in lipid rafts facilitates antigen presentation. Nat. Immunol. 1: 156162

48. Hoessli DC, llangumaran S, Soltermann A, Robinson PJ, Borisch B and Nasir UdD (2000) Signaling through sphingolipid microdomains of the plasma membrane: the concept of signaling platform. Glycoconj. J. 17: 191-197

49. Nakamura S, Kozutsumi Y, Sun Y, Miyake Y, Fujita T and Kawasaki T (1996) Dual roles of sphingolipids in signaling of the escape from and onset of apoptosis in a mouse cytotoxic T-cell line, CTLL-2. J. Biol. Chem. 271: 12551257

50. Humpf HU, Schmelz EM, Meredith FI, Vesper H, Vales TR, Wang E, Menaldino DS, Liotta DC and Merrill Jr AH (1998). Acylation of naturally occurring and synthetic 1-deoxysphinganines by ceramide synthase. Formation of $\mathrm{N}$ palmitoyl-aminopentol produces a toxic metabolite of hydrolyzed fumonisin, AP1, and a new category of ceramide synthase inhibitor. J. Biol. Chem. 273: 19060-19064

51. Schmelz EM, Dombrink-Kurtzman MA, Roberts PC, Kozutsumi Y, Kawasaki T and Merrill Jr AH (1998) Induction of apoptosis by fumonisin B1 in HT29 cells is mediated by the accumulation of endogenous free sphingoid bases. Toxicol. Appl. Pharmacol. 148: 252-260

52. Hung WC, Chang HC and Chuang LY (1999) Activation of caspase-3-like proteases in apoptosis induced by sphingosine and other long-chain bases in Hep3B hepatoma cells. Biochem. J. 338: 161-166

53. Brenner B, Ferlinz K, Grassme H, Weller M, Koppenhoefer U, Dichgans J, Sandhoff K, Lang F and Gulbins E (1998) Fas/CD95/Apo-I activates the acidic sphingomyelinase via caspases. Cell Death Differ. 5: 29-37

54. De Maria R, Rippo MR, Schuchman EH and Testi R (1998). Acidic sphingomyelinase (ASM) is necessary for fas-induced GD3 ganglioside accumulation and efficient apoptosis of lymphoid cells. J. Exp. Med. 187: 897902

55. Tepper CG, Jayadev S, Liu B, Bielawska A, Wolff R, Yonehara S, Hannun YA and Seldin MF (1995) Role for ceramide as an endogenous mediator of Fasinduced cytotoxicity. Proc. Natl. Acad. Sci. USA 92: 8443-8447

56. Hsu SC, Wu CC, Luh TY, Chou CK, Han SH and Lai MZ (1998) Apoptotic signal of Fas is not mediated by ceramide. Blood 91: 2658-2663

57. Watts JD, Gu M, Polverino AJ, Patterson SD and Aebersold R (1997) Fasinduced apoptosis of $\mathrm{T}$ cells occurs independently of ceramide generation. Proc. Natl. Acad. Sci. USA 94: 7292-7296

58. Mueller CM and Scott DW (2000) Distinct molecular mechanisms of Fas resistance in murine B lymphoma cells. J. Immunol. 165: 1854-1862 